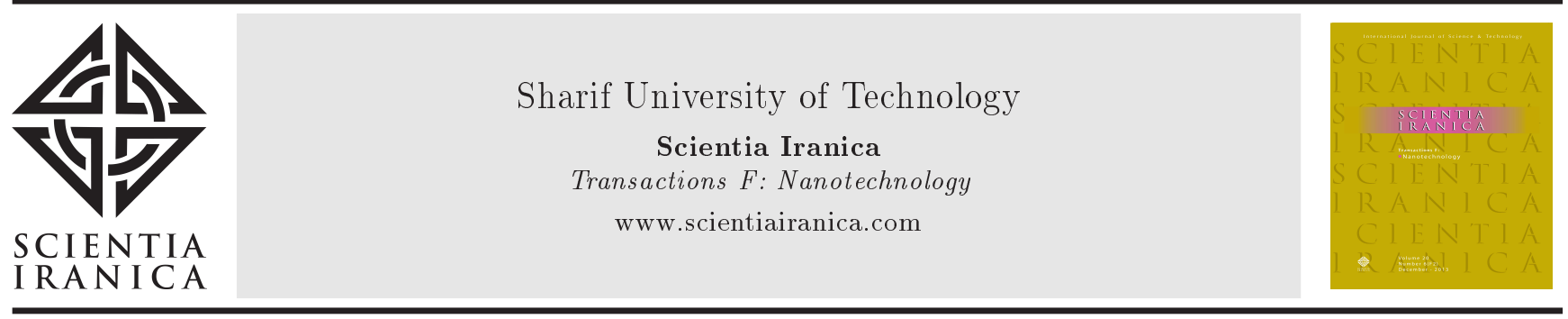

Research Note

\title{
The effect of pH on lateral size and surface chemistry of graphene oxide
}

\author{
F. Abbasi ${ }^{a}$, J. Karimi-Sabet ${ }^{\text {b,* }}$, C. Ghotbi ${ }^{a}$, Z. Abbasi ${ }^{a}$, S.A. Mousavi ${ }^{a}$ \\ and N. Amini ${ }^{a}$ \\ a. Department of Petroleum and Chemical Engineering, Sharif University of Technology, Tehran, Iran. \\ b. NFCRS, Nuclear Science and Technology Research Institute, Tehran, Iran. \\ Received 8 August 2016; received in revised form 26 October 2016; accepted 12 December 2016
}

\section{KEYWORDS \\ Attractive force; \\ Graphene oxide; \\ $\mathrm{pH}$; \\ Repulsive force.}

\begin{abstract}
In recent times, single-layer Graphene Oxide (GO) has been of intense interest to many researchers for a broad spectrum of applications. Size and surface charge of GO sheets are two key factors in most of these fields. These structural properties can be tuned by adjusting $\mathrm{pH}$ and manipulating the content of oxygen-containing groups. Furthermore, the $\mathrm{pH}$ value extremely affects the surface chemistry and functional groups of GO sheets, which are crucial in providing a high stable solution and chemical activity of particles. In this study, Graphene oxide is synthesized by the improved hummer method and characterized by XRD, AFM, FTIR, DLS, and zeta potential. pH value can be tuned through titration with $\mathrm{NaOH}$ and $\mathrm{HCl}$, and the effect of $\mathrm{pH}$ on $\mathrm{GO}$ dispersion stability and optical properties is investigated.
\end{abstract}

(C) 2017 Sharif University of Technology. All rights reserved.

\section{Introduction}

Graphene Oxide (GO) is a flat 2D layer of carbon atoms packed in a honeycomb lattice and is the basal building block in all graphitic materials with functional groups, including carboxylic acid, hydroxyl, epoxy, and carbonyl groups. GO, an exfoliation product of graphite oxide, has great influence on the nanoscience field $[1,2]$ because of its unique mechanical, thermal, and electrical properties $[3,4]$. According to the LerfKlinowski model, the carbonyl and carboxylic groups remain attached to the edges of GO sheet, while the hydroxyl and epoxy groups are found on the basal

\footnotetext{
*. Corresponding author.

E-mail addresses: fatemeabbasi@che.sharif.ir (F. Abbasi); j_karimi@alum.sharif.edu (J. Karimi-Sabet); ghotbi@sharif.edu (C. Ghotbi); z_abbasi@che.sharif.ir (Z. Abbasi); musavi@sharif.edu (S.A. Mousavi); negaramini86@gmail.com (N.Amini)
}

plane [5]. These groups endow GO with good processability and higher chemical activity than graphene in solution and enable it to be stably dispersed in water and chemically modified. In recent times, GO has been of intense interest to many researchers for a broad spectrum of applications and is widely used as a starting material to assemble specific graphenebased materials [6,7]. Therefore, the size of GO sheet and $\mathrm{C} / \mathrm{O}$ ratio largely influence the properties of $\mathrm{GO}$ derivatives. However, graphite oxide was first prepared by Brodie in 1859, by Staudenmaier [8] forty years later, and by Hammers and Offeman [9] in 1958; regrettably, the mechanism of GO formation and its structure is still not very clear due to the complexity of sheet size and functional groups, hindering the precise structure and property control of GO derivatives. The structure and properties of graphite oxide depend on synthesis method, degree of oxidation, and solvent type. Among broad applications of graphene oxide, a separation application is a relatively new topic since the graphene nanosheet has a rigid structure, which is 
impermeable to all molecules. GO sheet size, surface charge, and solution stability are all important factors in the separation performance. Therefore, in this research, we investigate the effect of $\mathrm{pH}$ on surface charge, particle size, and colloid stability of graphene oxide.

\section{Materials and method}

Graphite powders, sulfuric acid (95-97\%), phosphoric acid, hydrochloric acid (37\%), potassium persulfate, phosphorus pentoxide, hydrogen peroxide (30\%), potassium permanganate, ethanol, and sodium hydroxide were purchased from Merck.

GO was prepared using the improved Hummers method [10]. Briefly, $3 \mathrm{~g}$ of graphite powder was put into a solution of $\mathrm{H}_{2} \mathrm{SO}_{4}(98 \%, 12 \mathrm{~mL}), \mathrm{K}_{2} \mathrm{~S}_{2} \mathrm{O}_{8}$ $(2.5 \mathrm{~g})$, and $\mathrm{P}_{2} \mathrm{O}_{5}(2.5 \mathrm{~g})$ with continuous stirring for $4.5 \mathrm{hr}$ at $80^{\circ} \mathrm{C}$. After that, the mixture was cooled to room temperature, diluted with $0.5 \mathrm{~L}$ of water, and left overnight. Then, it was filtered to remove the residual acid and washed thoroughly with water. After drying at room temperature overnight, this pre-oxidized graphite was put into a 9:1 mixture of concentrated $\mathrm{H}_{2} \mathrm{SO}_{4} / \mathrm{H}_{3} \mathrm{PO}_{4}(360: 40 \mathrm{~mL})$, and then 18 gr of $\mathrm{KMnO}_{4}$ was added gradually while stirring. The reaction was then heated to $50^{\circ} \mathrm{C}$ and stirred for $12 \mathrm{~h}$. After that, the resulting mixture was cooled to room temperature and poured onto ice $(400 \mathrm{~mL})$ with $30 \%$ hydrogen peroxide $(3 \mathrm{~mL})$. Then, the mixture was centrifuged and the remaining solid material was washed several times with $200 \mathrm{~mL}$ of water and $200 \mathrm{~mL}$ of $30 \% \mathrm{HCl}$. The obtained solid was dried overnight at $50^{\circ} \mathrm{C}$ and dispersed in d-ionized water $(0.2 \mathrm{mg} / \mathrm{mL})$ using an ultrasonicator. The stable colloid had initial $\mathrm{pH}$ of about 3.74 , and this value can be tuned by $\mathrm{HCl}$ and $\mathrm{NaOH}$. The crystal structure of graphite oxide powder was characterized by the X-ray diffraction. XRD measurements were carried out by a XPERT-PRO diffractometer using the $\mathrm{Cu}$ $\mathrm{K} \alpha$ radiation wavelength $(\lambda=1.54 \AA)$ at $40 \mathrm{kV}$ and $40 \mathrm{~mA}$. The diffraction pattern was collected at room temperature in the range of $5^{\circ} \leq 2 \theta \leq 80^{\circ}$ with the step width of $0.0390^{\circ}$. The chemical bonds of graphite oxide powder were measured by Fourier Transform Infrared Spectroscopy in the range of $4000-1000 \mathrm{~cm}^{-1}$. Surface morphology and average particle size of GO sheets were observed using Atomic Force Microscopy (AFM, Vecco, noncontact (NC-AFM) mode, NCHR Si cantilevers) under tapping mode. To prepare samples for AFM imaging, $0.05 \mathrm{mg} / \mathrm{mL}$ GO suspension was first diluted 14 times. One drop of diluted suspension was deposited onto freshly cleaved quartz sheet and dried at room temperature. The GO surface charge and size distribution were measured by zeta potential meter (Molvern Zetasizer; ZEN3600 Model).

\section{Results and discussion}

XRD pattern was analyzed for the better understanding of GO structure. Based on XRD pattern, graphene shows a strong diffraction signal at $2 \theta=26.25^{\circ}$ which corresponds to a d-spacing of $3.4 \AA$. In contrast, GO powder shows a characteristic peak at $10.35^{\circ}$ corresponding to a d-spacing of $8.54 \AA$ (Figure 1). The average d-spacing from the first peak has been determined by Bragg's formula. Due to the oxidation process in which oxygen-containing functional groups, such as hydroxyl, carboxyl, and epoxide groups, are added to the graphene sheets, the interlayer spacing shifts from $3.4 \AA$ to $8.54 \AA$. The peak at 43 degrees corresponds to the turbostratic band of disordered carbon materials. The inter-layer d-spacing depends on the synthesis process (synthesis method and raw materials used) and the surrounding relative humidity [11]. Therefore, the value of $d$-spacing reported in the literature varies considerably. The inter-sheet interaction is decreased with increasing interlayer d-spacing; consequently, dispersion of GO sheets would be faster and easier, thus single layer GO can be obtained at low sonication time. Finally, high stable GO dispersion with the lowest structural defects would be achieved. The Crystallite size and average number of sheets can be calculated using Scherer's formula [12], which is equal to $85 \AA$ and 11 layers, respectively. The FTIR spectrum in Figure 2 indicates

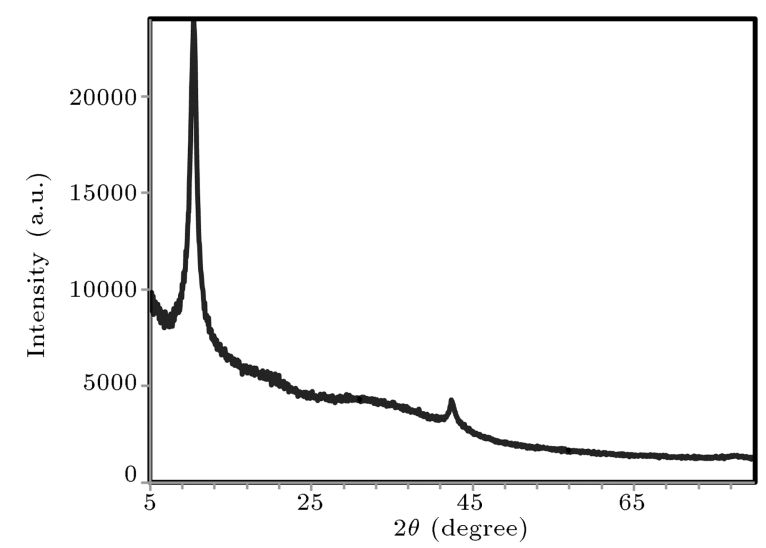

Figure 1. XRD spectrum of graphite oxide.

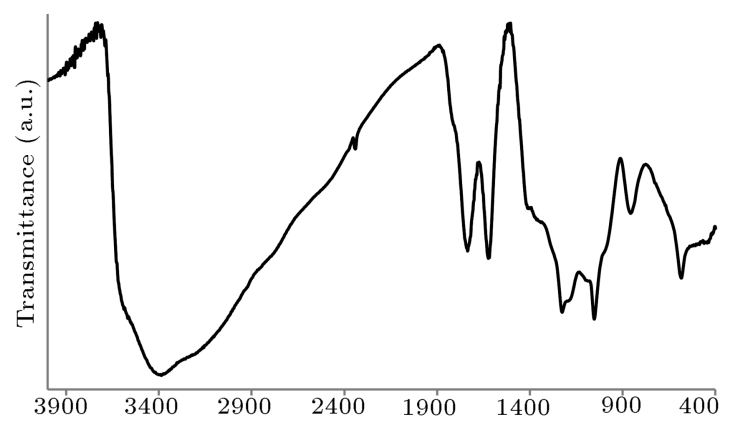

Figure 2. FTIR spectrum of GO nanosheet. 
the presence of hydroxyl groups on the basal plane $(\mathrm{OH}$ stretching at $3394.73 \mathrm{~cm}^{-1}$ ) and epoxy/ether groups $\left(\mathrm{C}-\mathrm{O}-\mathrm{C}\right.$ stretching at $1052 \mathrm{~cm}^{-1}$ ) as well as carboxyl groups $\left(\mathrm{C}=\mathrm{O}\right.$ stretching at $\left.1740 \mathrm{~cm}^{-1}\right)$. The spectrum shows peak at a wavenumber of $1620 \mathrm{~cm}^{-1}$ related to the expected deformation mode of $\mathrm{OH}$ group and at a $1224 \mathrm{~cm}^{-1}$ originated from the $\mathrm{C}=\mathrm{O}$ stretching vibrations of alkoxy. This means that the carboxylic acid groups were formed on the graphene surface. The bonds near 588 and 856 are most likely to be ascribed to in-plane and out-plane bending modes of $\mathrm{COO}^{-}$groups and benzene rings, respectively. These functional groups result in hydrophilicity, hydrogen bonding interactions between GO nano sheets and proper intersheet spacing, leading to the film structural stability and good mechanical strength, which are important in the many applications of GO films.

Atomic force microscopy demonstrates the flaky structure and topographical features of exfoliated GO sheets at various $\mathrm{pH}$ levels. The thickness $(<1 \mathrm{~nm})$ and lateral size of GO nanosheets can be clearly seen in the depth profile of Figure 3. In the chart, regions of higher depth correspond to the single-layer assembly of GO sheets. This might be originated from sample preparation, since we used the drop casting instead of spin coating method. The single-layer graphite oxide can be viewed as an unconventional type of soft material, and in contrast to hard colloidal particles, the single layer GO tends to bend and crease; this feature makes it difficult for multilayers to form.

GO suspensions are stable in the $\mathrm{pH}$ range of 311. Poor stability of the GO suspension was observed at both extremes of $\mathrm{pH}$ (less than $\mathrm{pH}$ of 2 and more than $\mathrm{pH}$ of 11) and relatively unstable after a week (Figure 4). After ultrasonicator, the graphite oxide was exfoliated onto several layers, and stable GO suspension was obtained. Figure 3 indicates that the small GO nano sheets with the size of 200-500 nm
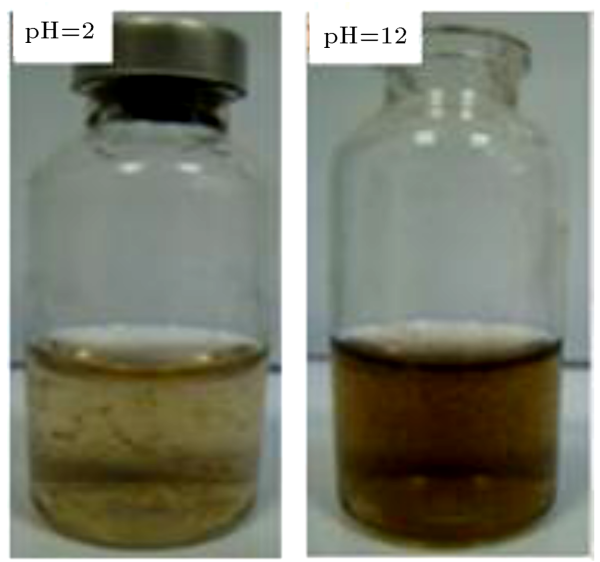

Figure 4. GO dispersion at 2 and $12 \mathrm{pH}$ after a week.
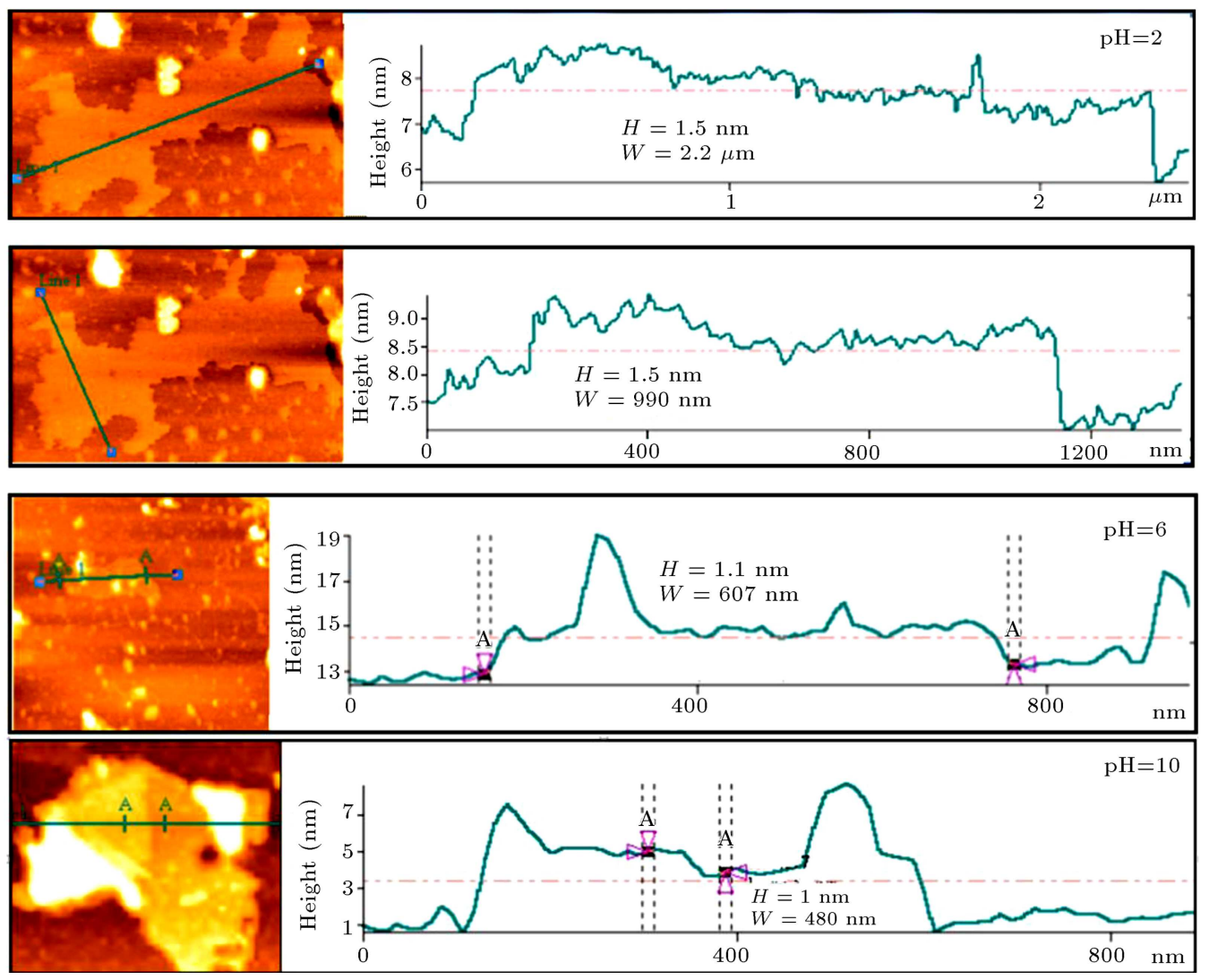

Figure 3. AFM image of a GO flake on freshly cleaved quartz. 

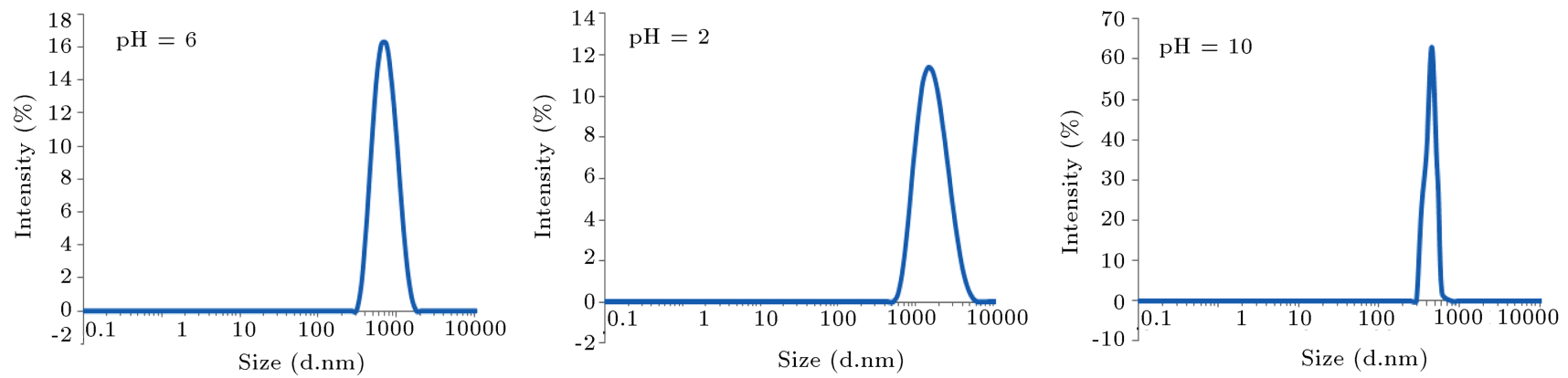

Figure 5. Particle size distribution of GO suspension at various $\mathrm{pH}$ levels.

can be observed in the alkaline environment, while the larger GO Nano sheets (GONs), whose sizes are distributed at about $500 \mathrm{~nm}-1.5 \mu \mathrm{m}$, tend to exist in the neutral and acidic conditions. At $\mathrm{pH}$ of 2 , the GO sheets agglomerate and their size sharply increases over 1.5 micron, with few sheets in the nanometric range. DLS is a technique that is well suited for estimating the size of spherical shaped particles, whereas GO has a planar structure and the DLS pattern of such a system could provide a convoluted result. It should be mentioned here that we do not attempt to estimate the average GO particle size; the aim of this analysis is to compare the relative change in the GO lateral size with $\mathrm{pH}$, which is shown in Figure 5 . The most chemically reactive portion of GO is the acidic carboxyl group and sheet size is largely dependent upon carboxyl groups on the GO nano sheet edge. Ionization of these groups in the acidic environment has a significant effect on the size of flakes. However, in the alkaline environment, the situation becomes complicated since both ionization and reduction of carboxylic groups occur. Of course, the reduction of hydroxyl groups is possible to occur in acidic environments. Zeta potential is an important factor in characterizing the stability of colloidal dispersions and surface charge. Our measurements show that zeta potential for the GO colloids is pH sensitive (Figure 6). Figure 6 depicts that change in the surface charge does not hold a linear relationship with the $\mathrm{pH}$.

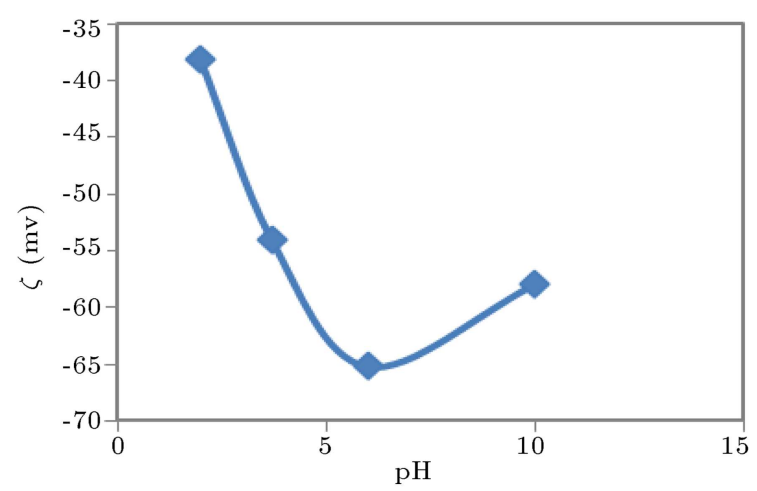

Figure 6. Zeta potential of GO suspension at various $\mathrm{pH}$ levels.
Generally, the density of ionized $-\mathrm{COO}^{-}$groups increases with the decrease of the face-to-edge ratio of GON; thereby, surface charge and electrostatic repulsion between GO nanosheets increase, enhancing colloidal stability eventually. In the acidic environment, with the decrease of $\mathrm{pH}$, the ionized $-\mathrm{COO}^{-}$groups are gradually protonated, leading to the increase in size, decrease in repulsion force, and the deposition of larger GONs at the lower $\mathrm{pH}$. By adding $\mathrm{NaOH}$, carboxyl groups are deprotonated, thus increasing zeta potential and decreasing GO sheet size. Adding more $\mathrm{NaOH}$, the $\mathrm{COOH}$ groups will be partly removed due to the counteract reaction, which also causes a precipitation of the large GONs from suspension at high $\mathrm{pH}$ (see Figure 4). The above results suggest a new route for precisely controlling the size of GONs in solution. Zeta potential measurements also reveal the change in the surface chemistry of GO sheets. With increasing $\mathrm{pH}$, zeta potential first reduces and then increases (Figure 6). The decrease of zeta from acidic to natural environment is caused by deprotonation of the $-\mathrm{COOH}$ groups, and an increase of it from alkaline to basic environment can be attributed to the reduction of them. Figure 7 illustrates a $0.05 \mathrm{mg} / \mathrm{ml} \mathrm{GO}$ dispersion at $\mathrm{pH}$ of 2 and 10 . As shown in this figure, GO dispersion colour changes from light at $\mathrm{pH}$ of 2 to dark brown at $\mathrm{pH}$ of 10 . According to the combination of the above discussions, it is considered that this colour transformation should be affected by the GO sheet

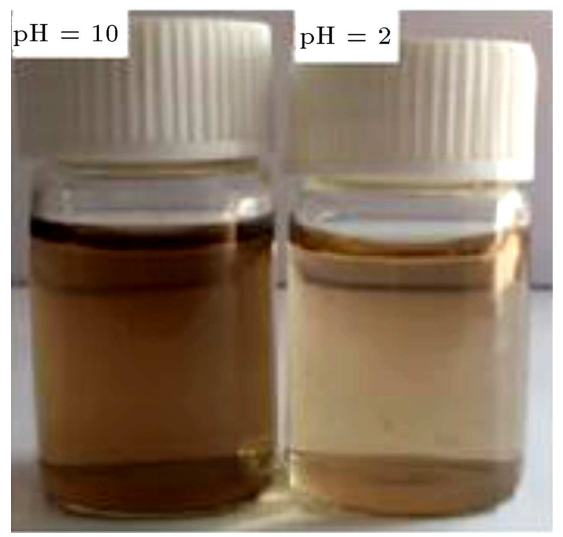

Figure 7. $0.05 \mathrm{mg} / \mathrm{ml}$ GO dispersion at various $\mathrm{pH}$ levels. 


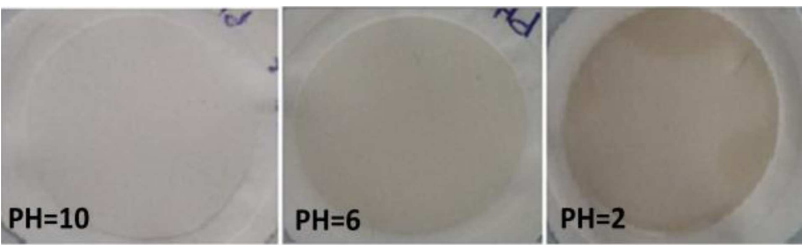

Figure 8. PA membrane at various pH levels.

size and surface chemistry. At higher pH, carboxyl groups are removed, thereby decreasing surface charge; eventually, GO structure tends to graphene one; this results in darker colour and poorer stability of GO dispersion at very high $\mathrm{pH}$.

In addition to DLS, AFM, and zeta potential analyses, the effect of $\mathrm{pH}$ on the GO sheet size can be realized by filtration. $3 \mathrm{ml}$ of $0.05 \mathrm{mg} / \mathrm{ml}$ aqueous $\mathrm{GO}$ dispersion prepared at $\mathrm{pH}$ of 2,6 , and 10 was filtered through polyamide (PA) membrane filter $(45 \mathrm{~mm}$, $0.45 \mu \mathrm{m}$ pore size; Whatman). It should be noted that the maximum attainable effective diameter of homesetup filter module was $3 \mathrm{~cm}$. As shown in Figure 8 , different surface membrane colors represent different amounts of graphene oxide on the membrane surface. In fact, transport of GO sheets across the PA membrane increases with $\mathrm{Ph}$, and this can be referred to the lateral size reduction of GO sheets with $\mathrm{pH}$. As previously mentioned, poor stability of the GO colloids was observed at both extremes of $\mathrm{pH}$ due to an decrease in the repulsive forces. In fact, the stability of GO suspensions depends on colloidal particle interaction. The GO suspension is stable if the electrostatic repulsion dominates and coagulation tendency decreases in this condition. On the other hand, particles tend to aggregate due to Van der Waals attractions, and the GO suspension would be irreversibly unstable if these attraction forces dominate. If both of these forces are significant, the GO suspension would be kinetically stable and their aggregation can be reversed by agitation. Electrostatic repulsion between the ionized carboxylic and phenol hydroxyl groups of graphene oxide forms a stable suspension in water. There are two fundamental interacting geometries between GONs: edge-to-edge and faceto-face. Due to highly anisotropic shape of GO particles, the suspension stability depends on the manner that GONs approach each other. Note that the scaling law of Van der Waals potential versus separation $\left(1 / d^{n}\right)$ depends on the geometry of the interacting bodies. The Van der Waals potential depends on the space between two atoms $\left(1 / d^{6}\right)$ and two chains $\left(1 / d^{5}\right)$ if two flat sheets approach together in an edge-to-edge manner. For the case of face-to-face attraction, their Van der Waals potential scales with $1 / d^{2}$. For the former case, the attraction forces rapidly decrease with separation distance; thus, the electrostatic repulsion makes a stable suspension. For the face-to-face one, the suspension usually forms flocculation during storage since both electrostatic and Van der Waals forces are significant. When two GO sheets with very different sizes are brought together, the face-to-face attraction will be dominant; therefore, the repulsive potential has a minimum value, and GO suspension would be unstable.

These findings are useful in the fabrication of graphene oxide-based composites and membranes from aqueous processing methods. Surface charges of singlelayered GO sheets undoubtedly have a great impact on the lamellar structure, and the effects of repulsion force and ionic screening on the GO films must be considered.

\section{Conclusion}

Effect of $\mathrm{pH}$ on GO size and surface chemistry was investigated in this paper. The reduction and protonation (deprotonation) of the carboxyl groups on the GO sheets are responsible for the variation in surface charge and lateral size of GO sheets. The zeta potential of the GO suspension with various $\mathrm{pH}$ levels reveals that its surface charge density strongly depends on $\mathrm{pH}$. Repulsion between the negatively charged sheets, proportional to surface charge, increases in natural and alkaline environments. However, change in the surface charge and repulsion force does not hold a linear relationship with the $\mathrm{pH}$. This is caused by reduced carboxyl groups of GONs at higher $\mathrm{pH}$. In acidic environment, protonation of carboxyl groups reduces the negative charge on the sheets, thereby decreasing electrostatic repulsion between them, eventually resulting in the agglomeration of the sheets at $\mathrm{pH}$ less than 2 . De-protonation and reduction of carboxyl groups can occur in natural, alkaline and basic environments. The former leads to the increase of repulsion force, whereas the latter weakens it. GO dispersions were stable in the $\mathrm{pH}$ range of $4-11$ and unstable at both extremes of the $\mathrm{pH}$ (2 and 12) due to the increase in attractive force and decrease in the repulsive forces, which are originated from protonation or reduction of carboxyl groups. Therefore, size and surface chemistry of GO sheet and GO dispersion stability are drastically influenced by $\mathrm{pH}$.

\section{References}

1. Zhu, Y., Murali, S., Cai, W., Li, X., Suk, J.W., Potts, J.R. and Ruoff, R.S. "Graphene and graphene oxide: synthesis, properties, and applications", Advanced $M a-$ terials, 22(35), pp. 3906-3924 (2010).

2. Chen, D., Feng, H. and Li, J. "Graphene oxide: preparation, functionalization, and electrochemical applications", Chemical Reviews, 112(11), pp. 6027-6053 (2012).

3. Heersche, H.B., Herrero, P.J., Oostinga, J.B., Vandersypen, M.K. and Morpurgo, A.F. "Induced superconductivity in graphene", Solid State Communications, 143(1), pp. 72-76 (2007). 
4. Balandin, A.A., Ghosh, S., Bao, W., Calizo, I., Teweldebrhan, D., Miao, F. and Lau, C.N. "Superior thermal conductivity of single-layer graphene", Nano Letters, 8(3), pp. 902-907 (2008).

5. Dreyer, D.R., SP ark "CW Bielawski and RS Ruoff", Chem. Soc. Rev., 39, p. 228 (2010).

6. Luo, B., Liu, S. and Zhi, L. "Chemical approaches toward graphene-based nanomaterials and their applications in energy-related areas", Small, 8(5), pp. 630646 (2012).

7. Chen, C., Yang, Q.H., Yang, Y., Lv, W., Wen, Y., Hou, P.X., Wang, M. and Cheng, H.M. "Self-assembled free-standing graphite oxide membrane", Advanced Materials, 21(29), pp. 3007-3011 (2009).

8. Staudenmaier, L. "Verfahren zur darstellung der graphitsäure", Berichte der Deutschen Chemischen Gesellschaft, 31(2), pp. 1481-1487 (1898).

9. Hummers Jr, W.S. and Offeman, R.E. "Preparation of graphitic oxide", Journal of the American Chemical Society, 80(6), pp. 1339-1339 (1958).

10. Marcano, D.C., Kosynkin, D.V., Berlin, J.M., Sinitskii, A., Sun, S., Slesarev, A., Alemany, L.B., Lu, W. and Tour, J.M. "Improved synthesis of graphene oxide", ACS Nano, 4(8), pp. 4806-4814 (2010).

11. Nair, R.R., Wu, H.A., Jayaram, P.N., Grigorieva, I.V. and Geim, A.K. "Unimpeded permeation of water through helium-leak-tight graphene-based membranes", Science, 335(6067), pp. 442-444 (2012).

12. Huh, S.H. "X-ray diffraction of multi-layer graphenes: Instant measurement and determination of the number of layers", Carbon, 78, pp. 617-621 (2014).

\section{Biographies}

Fateme Abbasi was born in 1991 in Iran. She received her BS degree from Department of Chemical Engineering at Petroleum University of Technology, Ahvaz, Iran, in 2013, and MS degree in Chemical Engineering in Department of Chemical Engineering at Sharif University of Technology, Tehran, Iran, in 2015. She is currently the Chemical Engineering PhD student at Sharif University of Technology. Her current research interests are in the areas of membrane and nanoparticle preparation.

Javad Karimi-Sabet was born in Iran in 1973. He received his BS and MS degrees in Chemical Engineering from Tehran University. Later on, he received his $\mathrm{PhD}$ degree in Chemical Engineering from the Department of Chemical Engineering at Sharif University of Technology. Currently, he works in the
Nuclear Science and Technology Research Institute as a researcher. His research work focuses on stable isotope separation. He has published more than 30 research articles and has more than 50 international conference papers.

Cyrus Ghotbi was born in 1956 in Tehran, Iran. He obtained his BS degree from the Department of Chemical Engineering at Sharif University of Technology, Tehran, Iran in 1978, and MS and PhD degrees in Petroleum Engineering from the French Institute of Petroleum (FIP), Paris in 1981 and 1984, respectively. He is presently a Professor of Chemical Engineering at Sharif University of Technology, Tehran, Iran. His research interests include thermodynamics, separation processes, and petroleum characterization. Professor Ghotbi is the author of about 110 papers published in international journals, and about 90 papers presented in international and national conferences.

Zeinab Abbasi was born in 1991 in Iran. She received her BS degree from the Department of Chemical Engineering at Petroleum University of Technology, Ahvaz, Iran in 2013, and MS degree in Chemical Engineering from the Department of Chemical Engineering at Sharif University of Technology, Tehran, Iran in 2015. She is currently a PhD student of Chemical Engineering at Sharif University of Technology. Her research interests are in the areas of transport phenomena, magnetic field, and separation process.

Seyyed Abbas Mousavi received his BS degree in Chemical Engineering from Sharif University of Technology, Tehran, Iran in 1999, and his MS and PhD degrees in Polymer Engineering from the same University in 2001 and 2007, respectively. His current research interests are in the areas of membrane preparation for gas separation, water treatment, waste water treatment, and micro and ultrafiltration processes. Now, he is an Associate Professor of Polymer Engineering at Sharif University of Technology.

Negar Amini was born in Iran in 1989. She received her BS degree from the Department of Chemical Engineering at Kermanshah University of Technology, Kermanshah, Iran in 2012, and MS degree in Chemical Engineering at the Department of Chemical Engineering at Sharif University of Technology, Tehran, Iran in 2015. Her current research focuses on nanoparticle preparation. 